
\title{
28 Research Suare \\ Ultrastructure of Anterior Lens Capsule and Epithelium in High-Myopic Cataracts
}

\section{Yingbin Wang}

Provincial Clinical College, Fujian Medical University

Wenjie Wu ( $\nabla$ wenjie_wu@foxmail.com )

Fujian Provincial Hospital

\section{Yajing Cai}

Provincial Clinical College, Fujian Medical University

\section{Mengting Yu}

Provincial Clinical College, Fujian Medical University

\section{Xiurong Zhong}

Fujian Medical University

\section{Minxia Wu}

Fujian Medical University

\section{Research Article}

Keywords: Cataract, High myopia, Electron microscopy, Lens capsule, Lens epithelium

Posted Date: April 14th, 2021

DOI: https://doi.org/10.21203/rs.3.rs-412523/v1

License: (c) (1) This work is licensed under a Creative Commons Attribution 4.0 International License. Read Full License 


\section{Abstract}

BACKGROUND: The mechanism of high-myopic cataract (HMC) is not fully understood yet. The ultrastructure of anterior lens capsule (aLC) and lens epithelial cells (LECs) may provide some clues to this issue.

METHODS: Capsulorhexis samples from 10 eyes with $\mathrm{HMC}$ and 10 eyes with age-related cataract (ARC) were analyzed for morphologic characteristics using transmission electron microscopy (TEM).

RESULTS: Several common pathologic disorders of LECs, including pleomorphic cellular structure, extensive intracellular vacuoles, swollen organelles, degeneration and necrotic lesions, could be observed in both HMC group and ARC group, to a similar extent. Specimens of HMC group disclosed remarkable extracellular spaces, interdigitation of LECs as well as protuberances of aLC towards LECs.

CONCLUSIONS: The ultrastructure of aLC and LECs in HMC demonstrated some specific pathologic disorders in comparison with ARC, which might play a role in the formation of cataract in high myopes.

\section{Background}

There is an unprecedented epidemic of short-sightedness in many parts of the world, especially in East Asia [1]. Myopia and high myopia are predicted to impact $49.8 \%$ and $9.8 \%$ of global population respectively by 2050 , emerging as an incremental worldwide public health issue [2]. This phenomenon foreshadows a considerable increase in complications of pathologic myopia, for example, the highmyopic cataract (HMC). Relationship between cataract (posterior subcapsular cataract and nuclear cataract) and high myopia is well established [3]. Nevertheless, mechanism of HMC is not completely understood yet.

It is well known that anterior lens capsule (aLC) and lens epithelial cells (LECs) not only play a physical defense role, but also serve as a regulatory barrier between aqueous humor and lens fibers to guarantee the transparency of crystalline lens [4,5]. We performed morphology analysis of aLCs and LECs obtained from routine cataract surgeries by transmission electron microscope (TEM) to further investigate the underlying etiology of HMC. To the best of our knowledge, no research has focused on this topic so far.

\section{Methods}

\section{Patients}

This electron microscopic investigation was conducted in Fujian Provincial Hospital and Fujian Medical University. Twenty consecutive patients diagnosed as ARC (10 patients) or HMC (10 patients) were recruited. We included ultra-high myopes whose axial length exceeding $29 \mathrm{~mm}$. The preoperative best corrected vision acuity was worse than 0.4 logMAR and cataract surgical intervention was required. Every patient underwent a detailed history inquiry and a comprehensive ophthalmic examination (Table 1). 
Exclusion criteria included: (1) systemic diseases such as diabetes mellitus and hypertension; (2) ocular disorders such as ocular infection, uveitis, glaucoma, and retinopathy; (3) history of ocular trauma or intraocular surgery; (4) any form of steroid usage. Written informed consents to participate in this study were obtained from all patients before the operation. 
Table 1

Information of patients included in this study

\begin{tabular}{|c|c|c|c|c|c|c|}
\hline Group & No. & Age & Gender & $\begin{array}{l}\text { Baseline BCVA } \\
\text { (logMAR) }\end{array}$ & $\begin{array}{l}\text { Axial length } \\
(\mathrm{mm})\end{array}$ & Nuclear density \\
\hline \multirow[t]{10}{*}{ ARC group } & 1 & 61 & $M$ & $\mathrm{CF} / 30 \mathrm{~cm}$ & 24.50 & IV \\
\hline & 2 & 61 & $\mathrm{~F}$ & 0.7 & 23.11 & ૫ \\
\hline & 3 & 61 & $\mathrm{~F}$ & 0.4 & 22.88 & प \\
\hline & 4 & 62 & $\mathrm{~F}$ & 0.4 & 22.86 & Q \\
\hline & 5 & 64 & $\mathrm{~F}$ & 0.6 & 23.20 & प \\
\hline & 6 & 65 & $\mathrm{~F}$ & 0.6 & 23.24 & प \\
\hline & 7 & 70 & M & 0.7 & 22.23 & IV \\
\hline & 8 & 72 & $\mathrm{~F}$ & 0.4 & 24.13 & प \\
\hline & 9 & 75 & $M$ & 0.5 & 23.13 & प \\
\hline & 10 & 76 & $\mathrm{~F}$ & 0.6 & 22.89 & Q \\
\hline \multirow[t]{10}{*}{ HMC group } & 11 & 46 & $\mathrm{~F}$ & 0.6 & 29.00 & ૫ \\
\hline & 12 & 48 & $\mathrm{~F}$ & 0.6 & 30.30 & ૫ \\
\hline & 13 & 52 & M & 0.6 & 30.71 & IV \\
\hline & 14 & 53 & $\mathrm{~F}$ & $\mathrm{CF} / 30 \mathrm{~cm}$ & 30.72 & IV \\
\hline & 15 & 59 & M & $\mathrm{CF} / 30 \mathrm{~cm}$ & 30.58 & V \\
\hline & 16 & 66 & $\mathrm{~F}$ & 1.0 & 32.28 & प \\
\hline & 17 & 68 & $M$ & $\mathrm{CF} / 1 \mathrm{~m}$ & 30.02 & IV \\
\hline & 18 & 71 & $\mathrm{~F}$ & 0.7 & 30.17 & Q \\
\hline & 19 & 74 & $\mathrm{~F}$ & $\mathrm{CF} / 1 \mathrm{~m}$ & 30.58 & IV \\
\hline & 20 & 80 & $\mathrm{~F}$ & 0.9 & 31.08 & IV \\
\hline \multicolumn{7}{|c|}{ BCVA: best corrected visual acuity; } \\
\hline \multicolumn{7}{|c|}{ Nuclear density: according to the Emery and Little classification; } \\
\hline \multicolumn{7}{|c|}{ ARC: age-related cataract; } \\
\hline
\end{tabular}


All tissue collection complied with the Guidelines of the Helsinki Declaration and was approved by the Medical Ethics Committee of Fujian Provincial Hospital (approval number: K2020-05-012). A 5-5.5mm circle of central aLC was obtained by routine continuous curvilinear capsulorhexis during uneventful phacoemulsification surgery and was fixed immediately for subsequent experiment.

\section{TEM Process}

Researchers of the Electron Microscope Laboratory of Fujian Medical University had no information about the subjects. Once the $5-5.5 \mathrm{~mm}$ circular specimens were gained, they were fixed in $3 \%$ glutaraldehyde- $1.5 \%$ paraformaldehyde- $0.1 \mathrm{M}$ phosphate buffer solution $(\mathrm{pH} 7.2)$ under $5^{\circ} \mathrm{C}$ for more than $48 \mathrm{~h}$ and post-fixed in $1 \%$ osmium tetroxide- $1.5 \%$ potassium ferrocyanide under $4^{\circ} \mathrm{C}$ for $2 \mathrm{~h}$. These two steps of fixation were respectively followed by rinse using $0.1 \mathrm{M}$ phosphate buffer solution $(\mathrm{pH} 7.2)$ for over 3 times. After dehydrated in increasing concentrations of ethanol and acetone ( $50 \%$ ethanol for $10 \mathrm{~min}, 70 \%$ ethanol-saturated uranyl acetate under $4^{\circ} \mathrm{C}$ overnight, $90 \%$ ethanol for $10 \mathrm{~min}, 90 \%$ ethanolacetone for $10 \mathrm{~min}, 90 \%$ acetone for $10 \mathrm{~min}$, anhydrous acetone for $10 \mathrm{~min}$ and 3 times), the specimens were embedded in epoxy resin 618. Ultrathin sections $(90-100 \mathrm{~nm})$ of the tissue blocks were made on the Ultramicrotome Leica EM UC7. Stained with uranyl acetate and lead citrate, the sections were examined under a FEI TECNAI transmission electron microscope. Two investigators independently examined and assessed all micrographs for the pathologic disorders. A third investigator would be asked for evaluation if there existed disagreement.

\section{Results}

Ten patients with $\mathrm{HMC}$ (aged $61.70 \pm 11.14$ years, axial length: $30.54 \pm 0.79 \mathrm{~mm}$ ) and ten patients with ARC (aged $66.7 \pm 5.69$ years, axial length: $23.22 \pm 0.62 \mathrm{~mm}$ ) were recruited in this study from July 2019 to June 2020 (shown in Table 1). We were not able to associate patients' age or nuclear density with TEM pathologic changes due to small sample size.

Relatively normal segments of aLC and LECs were occasionally observed in ARC group, which consisted of homogenous-appearing basement membrane and regular single-layered cobble-shaped cells with round nuclei, in absence of degeneration or necrosis (shown in Fig. 1). By contrast, the relatively normal architecture was not observed in HMC group.

Several pathological alterations could be seen in both HMC group and ARC group, to a similar extent: (1) LECs were altered from cuboidal shape to pleomorphic shape, with extensive intracellular vacuolization of cytoplasm and edema of mitochondria, endoplasmic reticulum as well as other undefined organelles (shown in Fig. 2a, 2b, 2c). (2) There existed degeneration and necrotic lesions of LECs: the degenerated cells, with remarkable aforementioned pathological changes, showed much lower electron density than the normal ones (shown in Fig. 2b); the necrotic epitheliums possessed ruptured cytomembrane and swollen nonfunctional organelles (shown in Fig. 3). Besides, apoptosis was discovered in neither HMC group nor ARC group. (3) Less frequent disorders comprised an increase of lysosomes inside some LECs and evidently flattened LECs with heavy-dyed nuclei (shown in Fig. 2b, 2d). 
According to our observation, specimens of HMC group disclosed more obvious extracellular vacuolization, placed between the lateral margins of adjacent LECs or between the basal lamina and LECs, varying from less than $1 \mu \mathrm{m}$ to over $10 \mu \mathrm{m}$ (shown in Fig. 4). Location of extracellular spaces varied among different specimens. In Patient 12, 13, 16, 17 and 20, most of the spaces were distributed close to epithelium-basal lamina border (shown in Fig. 4a). In Patient 15,18, they were mostly located close to the apical surface (shown in Fig. 4b). While in Patient 11,14,19, these two distribution manners could both be seen. A great portion of the extracellular spaces in $\mathrm{HMC}$ specimens was embedded with debris materials, $0.1-1 \mu \mathrm{m}$ in diameter (shown in Fig. 4b, 4d). Some were amorphous, some contained cristae-like fragments or microgranules and others possessed visible outer membrane. In some sections of the HMC specimens, concomitant with the extracellular vacuolization were multiple cell membrane folds, interdigitating with neighboring LECs (shown in Fig. 4c, 4d). The interdigitation could seldom be seen in ARC group. In addition, some LECs in HMC group showed branched cytoplasm which resembled fibroblasts (shown in Fig. 4a, 5a).

Another predominant disorder observed in five HMC specimens (Patient 12, 13, 15, 17, 18, aged 48, 52, 59, 68 and 71 respectively) was protuberances of basement membrane towards LECs. These protuberances were in different morphologic types, ranging from minimal bulge less than $1 \mu \mathrm{m}$ to long protuberance over $20 \mu \mathrm{m}$ (shown in Fig. 5). And there existed large extracellular spaces and debris around the polypoid protuberance. This disorder was obvious in Patient 13 and Patient 18, which was widespread over the whole basement membrane. In Patient 13, the electron density of some polypoid protuberances was much lower than normal basement membrane. A relatively transparent linear lesion extended from the polypoid protuberance towards anterior chamber in a vertical (shown in Fig. 5a, 5b, 5c). What's more, some segments of basal lamina in HMC group showed parallel laminal rarefication (shown in Fig. 6).

\section{Discussion}

In this study, we investigated the ultrastructure of aLC and LECs in HMC compared with ARC by TEM, to analyze the possible etiological mechanism of HMC. A lot of efforts have been devoted to explore the electron microscopic architecture of lens capsule since decades ago [6-15]. However, high-myopic cataracts haven't been involved so far.

A sheet of epitheliums is located between anterior lens capsule and fiber cells, with region-specific features $[4,16,17]$. Though the central epitheliums we collected from routine phacoemulsification surgery are considered essentially quiescent in terms of cell cycle, they drive active metabolic and transporting machinery to guarantee the homeostasis of the whole crystallin lens [4, 16-19]. By TEM, we discovered some common pathologic features in both HMC group and ARC group, to a similar extent, such as pleomorphic cellular structure, vacuolated cytoplasm, swollen organelles, as well as degeneration and necrotic lesions, which were also confirmed by previous studies of other cataractic types $[6-11,13,15]$. One of the prevalent hypotheses on cataractogenesis postulates that endogenous and exogenous reactive oxygen species (ROS) are the common pathway in various types of cataract development $[16,17$, 20-23]. LECs are considered as the initial target of oxidative stress damage [16, 17, 20-23]. Previous 
studies also demonstrated that with aging, the deposit of oxidized lens constituents together with the impairment of antioxidative function (which LECs play a role in) can bring about opacities in crystallin lens, resulting in caractogenesis [20-24]. Any disruption of morphologic structure might be implicated in dysfunction of LECs, which would accelerate cataract formation.

As to the underlying etiology of $\mathrm{HMC}$, one of the possible mechanisms might be that the crystallin lens of high myope suffers from higher concentration of oxygen, which is resulted from impaired physical and chemical barriers of vitreous body [3,24-26]. As a consequence, the surrounding ROS of crystallin lens add to the oxidative damage to LECs as well as the risk of cataract formation in high myopic eyes. What's more, a number of significantly changed metabolites in aqueous humor (where the anterior lens capsule was bathed in directly) of high myopes were identified by $\mathrm{Ji} \mathrm{YH}$ et al [27], but it is not verified yet whether or not the metabolic variations are involved in the morphological changes of LECs in high myopes.

In our study, there exist more extensive extracellular spaces and sometimes interdigitation between LECs in HMC group (shown in Fig. 4, 5). Similar extracellular vacuolization was also reported in a TEM study of LECs in presenile cataract [13]. Since all specimens in our experiment went through the same surgical procedure, we consider that the greater extracellular spaces in HMC group are irrelevant to the surgical stimulation. We proposed that the larger extracellular spaces might be the consequence of cell contraction and cytoskeletal reorganization. Firstly, an inverted microscopic research by Andjelic, et al demonstrated that LECs possessed the ability of contraction, and under non-specific stimulation, the intercellular width changed from barely noticeable to several microns [28]. Furthermore, larger extracellular spaces were also observed in LECs of a transgenic mouse model expressing Rho GTPase inhibitor compared with wild-type mouse LECs under TEM, while Rho GTPase was considered as a crucial point in actin cytoskeleton arrangement [29-31]. Researchers have also suggested that an inappropriate accumulation of transforming growth factor- $\beta$ (TGF- $\beta$ ) induced remodeling of lens cytoskeleton through Rho family of GTPases [30, 31]. In the meanwhile, Zhu XJ and his copartners found significant elevated TGF- $\beta 2$ concentration in the aqueous humor of highly myopic eyes, in proportion to axial length [32]. Therefore, it might be worthwhile to further explore the expression of TGF- $\beta$ and Rho GTPase in surgical capsule samples of HMC as well as their association with lens epithelial cytoskeleton. In addition, the interdigitation between LECs was also observed in cataracts with retinal pigmentosa and uveitis [7, 9]. Andjelic, et al also reported similar interdigitation between adjacent LECs at the basal side but they were not related to the type of cataract (the specific cataract types were not provided though) [6]. However, in our study, the interdigitation of LECs were rarely found in ARC group.

Another significant characteristic observed in five high-myopic specimens (Patient 12, 13, 15, 17, 18, aged $48,52,59,68$ and 71 respectively) is the protuberances of extracellular matrix (ECM) towards LECs (shown in Fig. 5). This phenomenon was not reported by Andjelic and colleagues, whose experiment focused on the LECs attachment to aLC in various cataract types by a combination of TEM, scanning electron microscopy and confocal microscopy [6]. However, Hawlina $\mathrm{M}$ et al discovered similar protuberances with degraded cellular materials inside at the epithelium-basal lamina border in $86.6 \%$ samples of intumescent cataract and in $34.3 \%$ samples of nuclear cataract [33]. In their opinion, the 
function of the protuberances was to capture degrading LECs debris and after that, new layer of epithelium would be deposited upon the gap space. Different from this study, we didn't find captured cellular debris in the protuberances, and we observed distorted epithelium with notable extracellular spaces upon the protuberances rather than newborn LECs (shown in Fig. 5). Richiert DM, et al and Zhang $\mathrm{XH}$, et al respectively observed elevated secretion of ECM components (fibronectin and laminin) in animal model LECs treated by TGF- $\beta$, which was proved to increase in aqueous humor of highly myopic eyes [34-36].Hence, the specific components and origin of the protuberances along epithelium-basal lamina border in $\mathrm{HMC}$ need further investigation.

There are several limitations in this study: the crucial ones are the small sample size and the qualitative description; what's more, our hypothesis about the etiology of extracellular spaces among LECs and protuberances in aLC cannot be merely established by morphologic observation, so immunohistochemistry and other confirmable methods are needed. Therefore, our next step will be expanding sample size and finding objective evidence to explain our observation of lens capsule in HMC.

\section{Conclusions}

Compared with ARC, the ultrastructure of aLC and LECs in HMC demonstrated some specific pathologic disorders, which might play a role in the formation of cataract in high myopes. As far as we know, no research has explored the electron microscopic structure of aLC and LECs in HMC yet.

\section{List Of Abbreviations}

aLC: anterior lens capsule

ARC: age-related cataract

HMC: high-myopic cataract

LECs: lens epithelial cells

TEM: transmission electron microscopy

\section{Declarations}

\section{Ethics approval and consent to participate:}

This study was conducted ethically in accordance with the guidelines of human studies and the Helsinki Declaration. It was approved and consented by the Medical Ethics Committee of Fujian Provincial Hospital (approval number: K2020-05-012).

\section{Consent for publication}


Not applicable.

\section{Availability of data and materials}

The data and materials generated and analysed during the current study are available from the corresponding author on reasonable request.

\section{Competing interests}

The authors declare that they have no competing interests.

\section{Funding}

This study was supported by Grant No.2019J01187 from Natural Science Foundation of Fujian Province, China.

\section{Authors' Contributions}

WJW, YBW and YJC designed the investigation; the surgeries were conducted by WJW. XRZ and MXW conducted the experiment; MTY, XRZ and MXW analyzed and interpreted the results; the paper was drafted by YBW and revised by WJW. All authors read and approved the final manuscript.

\section{Acknowledgements}

Not applicable.

\section{References}

1. Dolgin E. THE MYOPIA BOOM. Nature. 2015;519(7543):276-8.

2. Holden BA, Fricke TR, Wilson DA, Jong M, Naidoo KS, Sankaridurg P, et al. Global Prevalence of Myopia and High Myopia and Temporal Trends from 2000 through 2050. Ophthalmology. 2016;123(5):1036-42.

3. Haarman AEG, Enthoven CA, Tideman JWL, Tedja MS, Verhoeven VJM, Klaver CCW. The complications of myopia: a review and meta-analysis. Invest Ophthalmol Vis Sci. 2020;61(4):49.

4. Bhat SP. The Ocular Lens Epithelium. Biosci Rep. 2001;21(4):537-63.

5. Danysh BP, Duncan MK. The lens capsule. Exp Eye Res. 2009;88(2):151-64.

6. Andjelic S, Draslar K, Hvala A, Lopic N, Strancar J, Hawlina M. Anterior lens epithelial cells attachment to the basal lamina. Acta Ophthalmol. 2016;94(3):e183-8.

7. Andjelic S, Draslar K, Hvala A, Hawlina M. Anterior lens epithelium in cataract patients with retinitis pigmentosa - scanning and transmission electron microscopy study. Acta Ophthalmol. 2017;95(3):e212-e20. 
8. Sorkou KN, Manthou ME, Tsaousis KT, Brazitikos P, Tsinopoulos IT. Transmission electron microscopy study of undescribed material at the anterior lens capsule in exfoliation syndrome. Graefes Arch Clin Exp Ophthalmol. 2018;256(9):1631-7.

9. Tekin K, Ozdamar Erol Y, Inanc M, Sargon MF, Cakar Ozdal P, Berker N. Ultrastructural Analysis of the Anterior Lens Epithelium in Cataracts Associated with Uveitis. Ophthalmic Res. 2019:1-9.

10. Stunf S, Hvala A, Vidovic Valentincic N, Kraut A, Hawlina M. Ultrastructure of the anterior lens capsule and epithelium in cataracts associated with uveitis. Ophthalmic Res. 2012;48(1):12-21.

11. Tekin K, Erol YO, Inanc M, Sargon MF, Can CU, Polat S, et al. Electron microscopic evaluation of anterior lens epithelium in patients with idiopathic congenital cataract. Int Ophthalmol. 2018;38(5):2127-32.

12. Inanc M, Tekin K, Erol YO, Sargon MF, Koc M, Budakoglu O, et al. The ultrastructural alterations in the lens capsule and epithelium in eyes with traumatic white cataract. Int Ophthalmol. 2019;39: 47-53.

13. Wu J, Zhou JY, Ping XY, Xu XY, Cui YL, Yang H et al. Scanning and transmission electron microscopy study of anterior lens epithelium in presenile cataract. Int Ophthalmol. 2020;40: 1411-1418.

14. Sorkou KN, Manthou ME, Meditskou S, Ziakas N, Tsinopoulos IT. Exfoliation Fibrils within the Basement Membrane of Anterior Lens Capsule: A Transmission Electron Microscopy Study. Curr Eye Res. 2019;44: 882-886.

15. Sorkou KN, Manthou ME, SoultanaMeditskou, Ziakas N, Tsaousis KT, Tsinopoulos IT. Severe Abnormalities of Lens Epithelial Cells in Exfoliation Syndrome: A Transmission Electron Microscopy Study of Patients with Age-Related Cataract. Medicina (Kaunas). 2019;55: undefined.

16. Martinez G, de longh RU. The lens epithelium in ocular health and disease. Int J Biochem Cell Biol. 2010;42(12):1945-63.

17. Dahm R, van Marle J, Quinlan RA, Prescott AR, Vrensen GF. Homeostasis in the vertebrate lens: mechanisms of solute exchange. Philos Trans R Soc Lond B Biol Sci. 2011;366: 1265-77.

18. Candia OA. Electrolyte and fluid transport across corneal, conjunctival and lens epithelia. Exp Eye Res. 2004;78: 527-35.

19. Delamere NA, Tamiya S. Expression, regulation and function of $\mathrm{Na}, \mathrm{K}-\mathrm{ATP}$ ase in the lens. Prog Retin Eye Res. 2004;23: 593-615.

20. Berthoud VM, Beyer EC. Oxidative stress, lens gap junctions, and cataracts. Antioxid Redox Signal. 2009;11(2):339-53.

21. Nita M, Grzybowski A. The Role of the Reactive Oxygen Species and Oxidative Stress in the Pathomechanism of the Age-Related Ocular Diseases and Other Pathologies of the Anterior and Posterior Eye Segments in Adults. Oxid Med Cell Longev. 2016;2016:3164734.

22. Truscott RJ. Age-related nuclear cataract-oxidation is the key. Exp Eye Res. 2005;80(5):709-25.

23. Øsnes-Ringen 0 , Azqueta AO, Moe MC, Zetterström C, Røger M, Nicolaissen B et al. DNA damage in lens epithelium of cataract patients in vivo and ex vivo. Acta Ophthalmol. 2013;91:652-6. 
24. Shui YB, Holekamp NM, Kramer BC, Crowley JR, Wilkins MA, Chu F, et al. The gel state of the vitreous and ascorbate-dependent oxygen consumption: relationship to the etiology of nuclear cataracts. Arch Ophthalmol. 2009;127(4):475-82.

25. Beebe DC, Holekamp NM, Siegfried C, Shui YB. Vitreoretinal influences on lens function and cataract. Philos Trans R Soc Lond B Biol Sci. 2011;366(1568):1293-300.

26. Morita H FM, Tokoro T. A clinical study of the development of posterior vitreous detachment in high myopia. Retina. 1995;15(2):117-24.

27. Ji YH, Rao J, Rong XF, Lou S, Zheng Z, Lu Y. Metabolic characterization of human aqueous humor in relation to high myopia. Exp Eye Res. 2017;159:147-55.

28. Andjelic S, Zupancic G, Perovsek D, Hawlina M. Human anterior lens capsule epithelial cells contraction. Acta Ophthalmol. 2011;89(8):e645-53.

29. Maddala R, Deng PF, Costello JM, Wawrousek EF, Zigler JS, Rao VP. Impaired cytoskeletal organization and membrane integrity in lens fibers of a Rho GTPase functional knockout transgenic mouse. Lab Invest. 2004;84(6):679-92.

30. Korol A, Taiyab A, West-Mays JA. RhoA/ROCK Signaling Regulates TGFß-Induced EpithelialMesenchymal Transition of Lens Epithelial Cells through MRTF-A. Molecular Medicine. 2016;22(1):713-23.

31. Maddala R RV, Epstein DL, Rao V. Growth factor induced activation of Rho and Rac GTPases and actin cytoskeletal reorganization in human lens epithelial cells. Mol Vis. 2003;9:329-36.

32. Zhu XJ, Chen MJ, Zhang KK, Yang J, Lu Y. Elevated TGF-beta2 level in aqueous humor of cataract patients with high myopia: Potential risk factor for capsule contraction syndrome. J Cataract Refract Surg. 2016;42(2):232-8.

33. Hawlina M, Stunf S, Hvala A. Ultrastructure of anterior lens capsule of intumescent white cataract. Acta Ophthalmol. 2011;89(4):e367-70.

34. Zhu XJ, Chen MJ, Zhang KK, Yang J, Lu Y. Elevated TGF-beta2 level in aqueous humor of cataract patients with high myopia: Potential risk factor for capsule contraction syndrome. J Cataract Refract Surg. 2016;42(2):232-8.

35. Richiert DM, ME I. TGF- $\beta$ elicits fibronectin secretion and proliferation in cultured chick lens epithelial cells. Curr Eye Res. 1999;18(1):62-71.

36. Zhang XH, Sun HM, Yuan JQ. Extracellular matrix production of lens epithelial cells. J Cataract Refract Surg. 2001;27(8):1303-9.

\section{Figures}




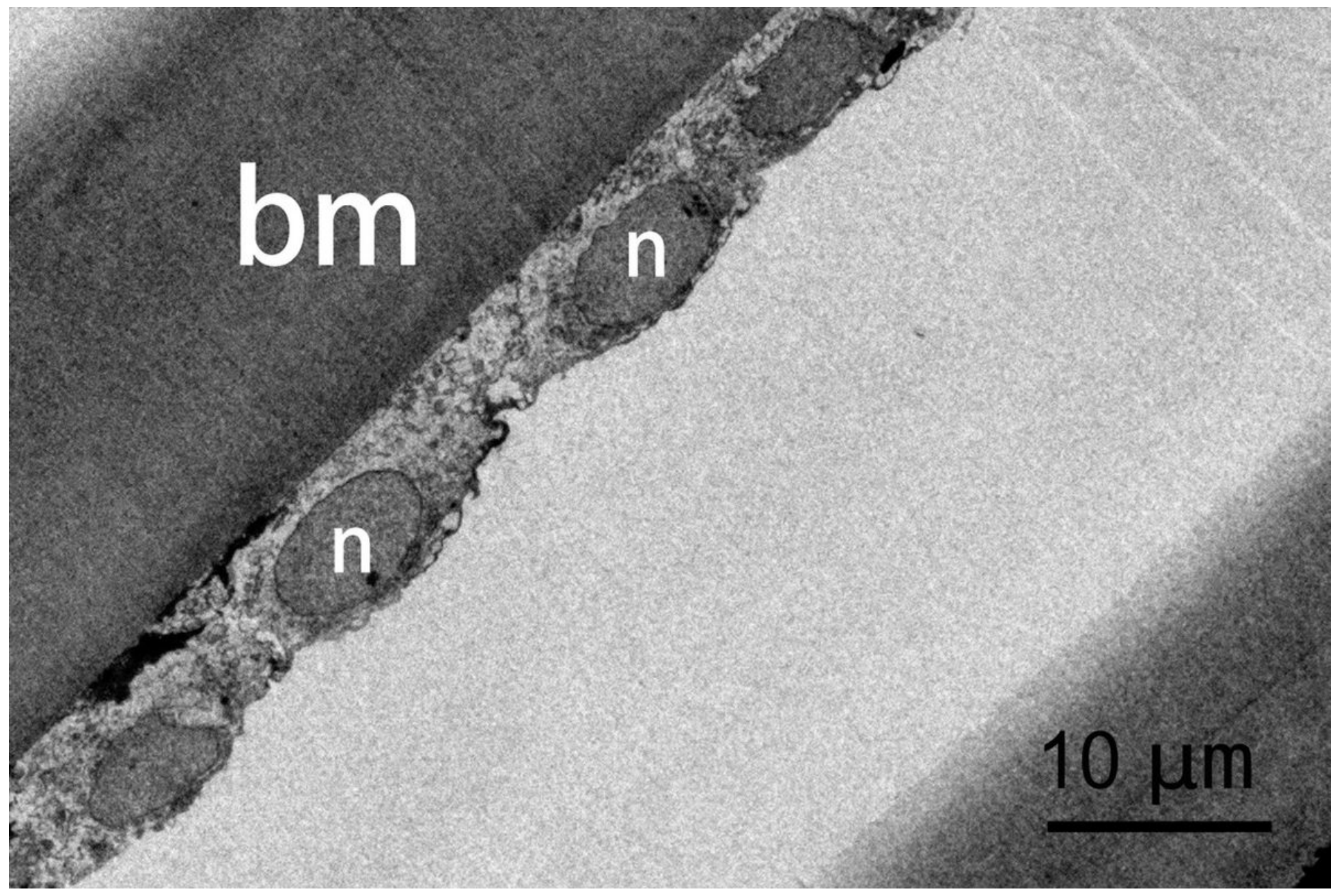

Figure 1

Transmission electron micrograph showing relatively normal aLC and LECs in a 61-year-old female patient with ARC (Patient 3). n, nucleus; bm, basal membrane (anterior lens capsule).
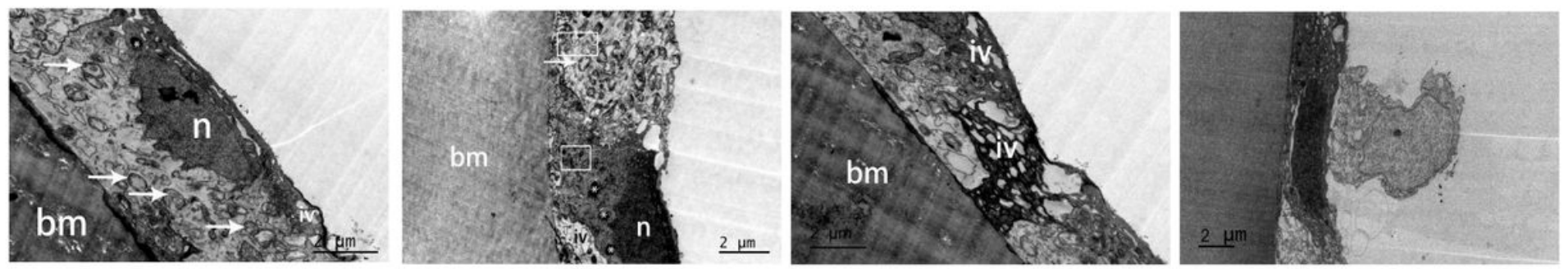

\section{Figure 2}

Intracellular pathologic disorders in both HMC and ARC groups (Fig. 2a: Patient 12; Fig. 2b: Patient 15; Fig. 2c: Patient 2; Fig. 2d Patient 7): shrunken nuclear membrane, swollen organelles, intracellular vacuoles. Fig. $2 d$ reveals a flat epithelial cell with an accidently detached epithelial cell. a. $n$, nucleus; bm, basal membrane (anterior lens capsule); iv, intracellular vacuole; arrow: mitochondrion; asterisk: lysosome. b. n, nucleus; bm, basal membrane (anterior lens capsule); arrow: mitochondrion; asterisk: 
lysosome; rectangle: endoplasmic reticulum. c. bm, basal membrane (anterior lens capsule); iv, intracellular vacuole.
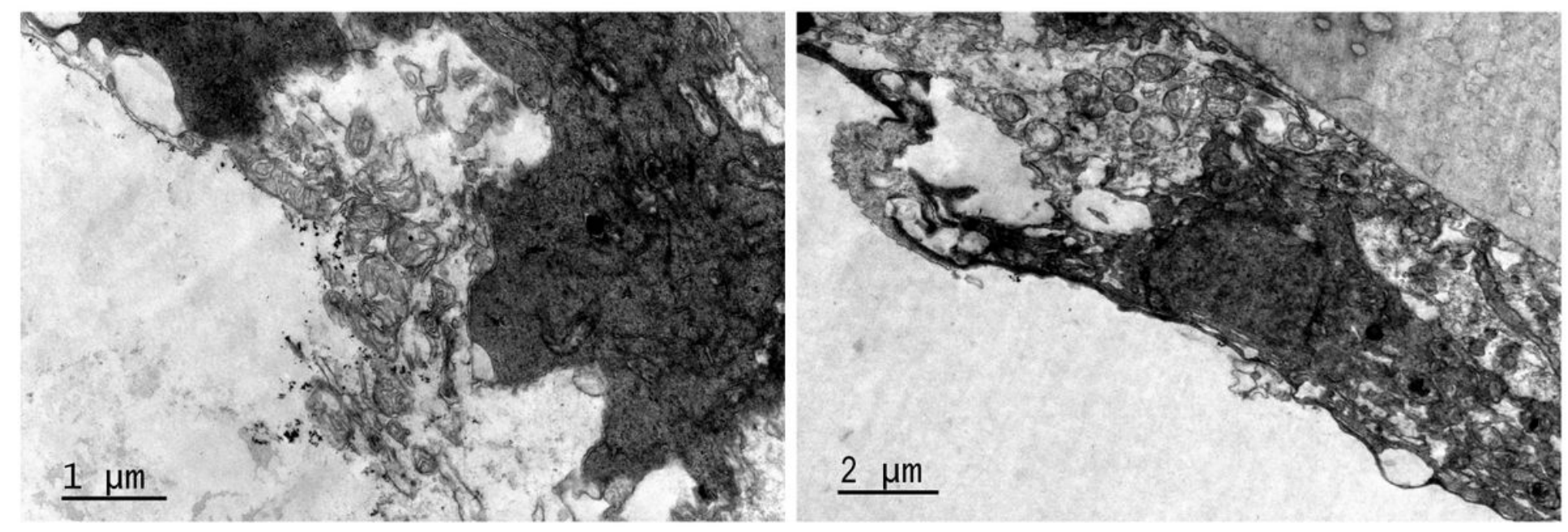

\section{Figure 3}

Necrotic lesions in a 61-year-old male patient with ARC (Fig. 3a: Patient 1) and a 46-year-old female patient with HMC (Fig. 3b: Patient 11).
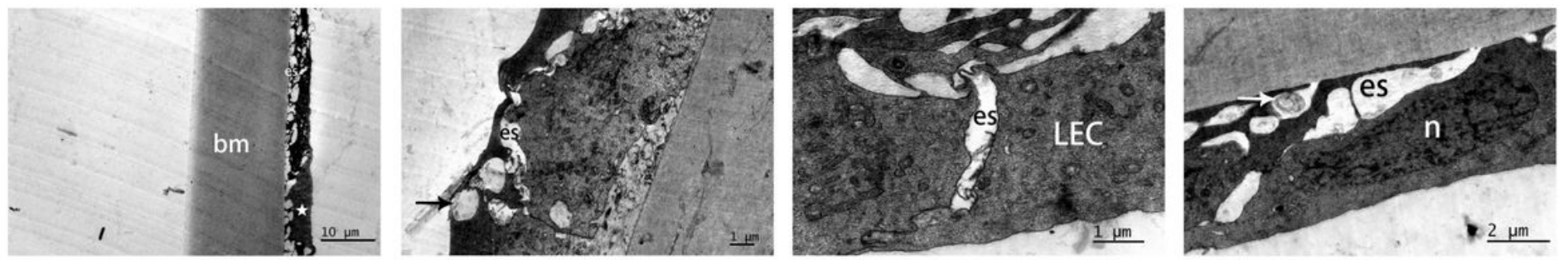

\section{Figure 4}

Significant extracellular spaces in HMC group (Fig. 4a: Patient 16; Fig. 4b: Patient 15; Fig. 4c, 4b: Patient 20). Fig. 4c and Fig. 4d demonstrate plenty of cellular interdigitations. a. bm, basal membrane (anterior lens capsule); es, extracellular space; star: fibroblast-like epithelium. b. es, extracellular space; arrow: debris material. c. LEC, lens epithelial cell; es, extracellular space. d. n, nucleus; es, extracellular space; arrow: debris material.
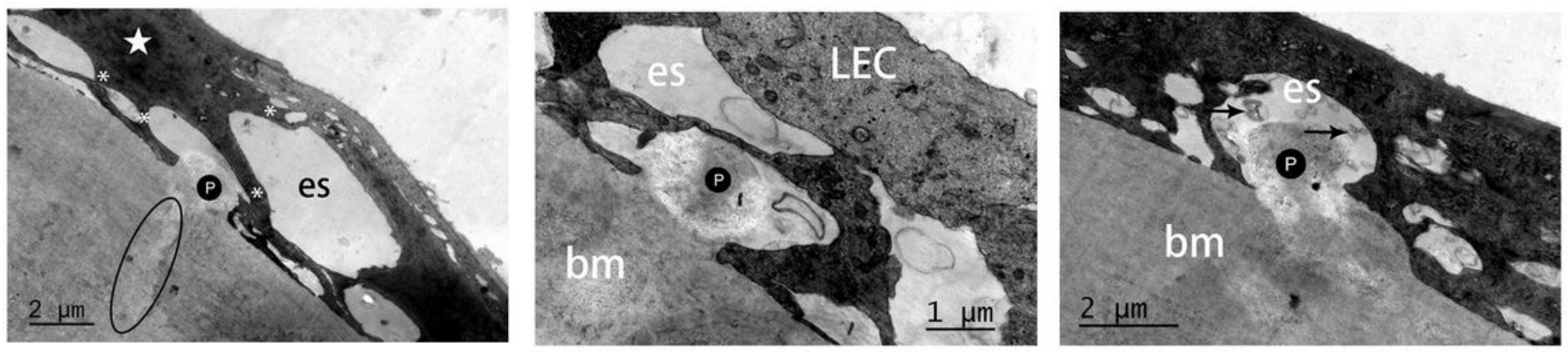


\section{Figure 5}

Protuberances of basal membrane, surrounded by pathologic extracellular spaces and debris materials in a 52-year-old male patient with HMC (Fig. 5a-5c: Patient 13). A relatively transparent zone extends from the polypoid protuberance in a vertical way (indicated by an ellipse). a. es, extracellular space; $\mathrm{p}$ : protuberance; star: fibroblast-like epithelium; asterisk: branched cytoplasm of fibroblast-like epithelium. b. LEC, lens epithelial cell; bm, basal membrane (anterior lens capsule); es, extracellular space; p: protuberance. c. bm, basal membrane (anterior lens capsule); es, extracellular space; p: protuberance; arrow: debris material.

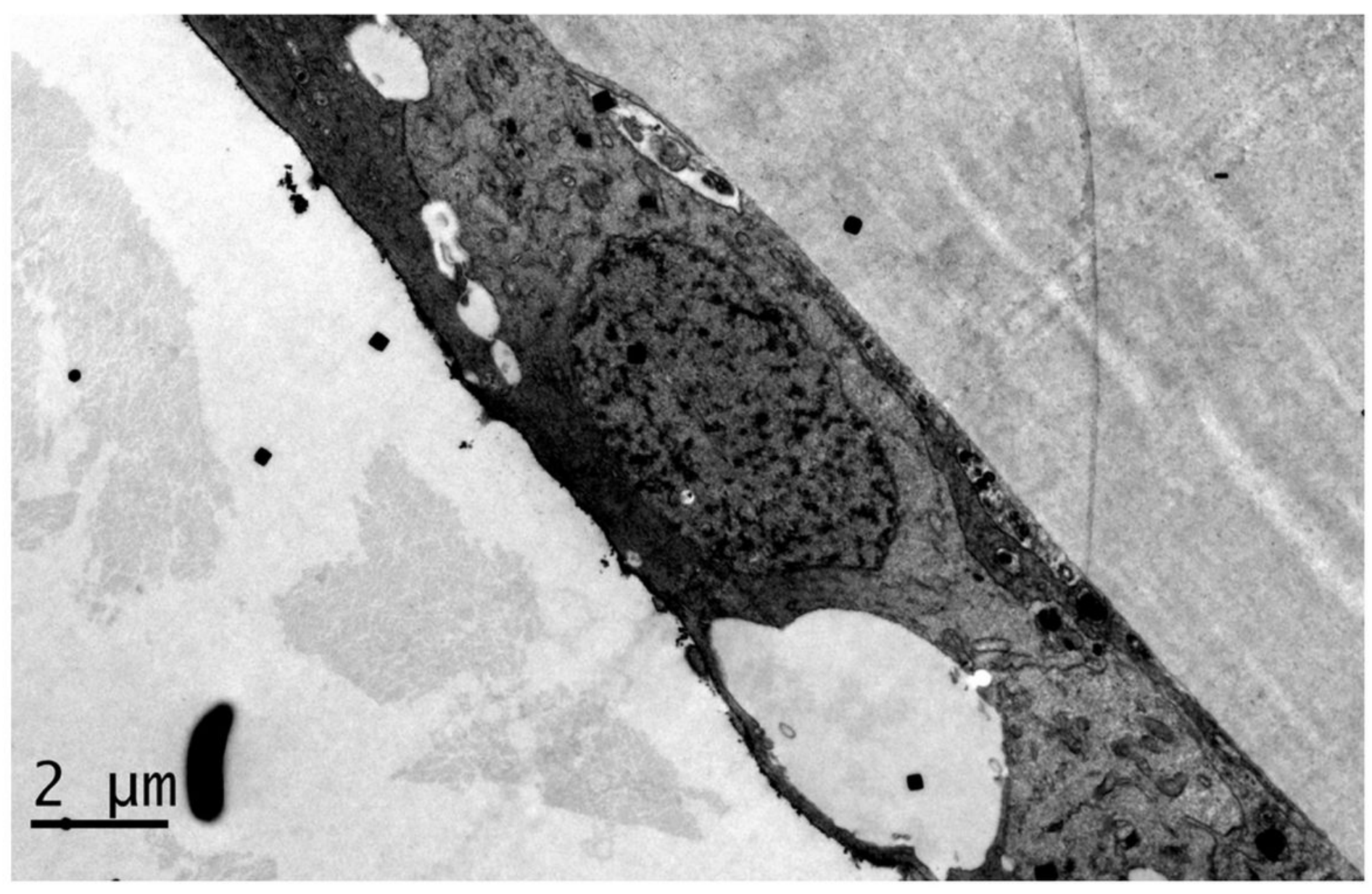

Figure 6

Segments of aLC in HMC group with parallel laminal rarefication (Patient 18). 